\title{
NÍVEIS DE LISINA EM RAÇÕES DE SUÍNOS EM TERMINAÇÃO: UM METANÁLISE PARA OTIMIZAR O DESEMPENHO
}

\author{
META-ANALYSIS OF LYSINE LEVELS USED IN DIETS FOR FINISHING SWINE IN \\ BRAZIL: RECOMMENDATIONS TO OPTIMIZE PERFORMANCE AND REDUCE COSTS
}

\author{
Portela, L.B. ${ }^{1 A}$; Siqueira, J.C. ${ }^{1{ }^{*}}$; Bomfim, M.A.D. ${ }^{1 C}$; Nascimento, D.C.N. ${ }^{2}$; Ribeiro, F.B. ${ }^{1 D}$; \\ Oliveira, F.L. ${ }^{1 E}$; Pereira, W.G. ${ }^{1 F}$ e Dos Santos, J.C. ${ }^{1 G}$
}

\begin{abstract}
${ }^{1}$ Centro de Ciências Agrárias e Ambientais. Universidade Federal do Maranhão (CCAA/UFMA). ChapadinhaMA.Brasil.Alarisssa_brandao_portela@yahoo.com.br; ${ }^{B *}$ jcsiqueira@ufma.br; ${ }^{C}$ madbomfim@yahoo.com.br; Dfelipebribeiro@yahoo.com; Eloiola93@gmail.com; Fwelligton.gomes88@gmail.com; Gjuliana.snt2@ gmail.com

${ }^{2}$ Departamento de Zootecnia. Centro de Ciências Agrárias. Universidade Federal do Piauí (CCA/UFPI). Teresina-PI. Brasil. daphinnec@yahoo.com.br
\end{abstract}

\section{Palavras chave adicionaIS}

Aminoácidos. Análise de custos. Suinocultura.

\section{RESUMO}

O objetivo do trabalho foi estimar os níveis de lisina (Lys) em rações de suínos em terminação, adequados para otimizar o desempenho e reduzir os custos com alimentação, utilizando a técnica da metanálise. Para isso foram utilizados dados de conversão alimentar (CA) de 16 experimentos dose-resposta realizados no Brasil, publicados entre os anos de 2000 e 2011, que avaliaram níveis crescentes de lisina na ração. Após convertidos para uma base comum, os dados de conversão alimentar dos trabalhos selecionados foram submetidos á análises de regressão considerando o nível de Lys como variável independente, utilizando os modelos exponencial, polinomial quadrático e Linear Response Plateau (LRP). Com base na equação exponencial, foi calculada a variação do custo com alimentação por $\mathrm{kg}$ de ganho de peso. Considerou-se o custo da ração basal (sem L-lisina $\mathrm{HCl}$ ) como sendo $\mathrm{R} \$ 0,70 / \mathrm{kg}$, e atribuiu-se o valor de 2,80 ( $\mathrm{g} / \mathrm{g})$ para a CA desejada. O nível ótimo de lisina total adequado para otimizar o desempenho, estimado a partir da utilização do modelo quadrático foi 1,036 \%, enquanto o nível ótimo estimado para reduzir os custos com alimentação e aumentar a margem foi $0,980 \%$ quando o $\mathrm{kg}$ de L-lisina $\mathrm{HCl}(78,5 \%)$ custou $\mathrm{R} \$ 4,71$. Por outro lado quando o $\mathrm{kg}$ de $\mathrm{L}$ -

\author{
AdDitionAL KEYWORDS \\ Amino acids. Cost analysis. Swine production.
}

lisina $\mathrm{HCl}(78,5 \%)$ aumentou para $\mathrm{R} \$ 7,85$, o nível estimado reduziu para $0,880 \%$. Para simular o custo com alimentação por $\mathrm{kg}$ de GP de suínos em crescimento/terminação propôs-se uma equação.

\section{SUMMARY}

The objective of the study was to determine optimal lysine levels (Lys) in diets for finishing pigs, suitable for optimize performance and reduce feed costs, using the meta-analysis technique. Feed conversion (FC) data from 16 dose-response experiments realized in Brazil (from 2000 to 2011), which evaluated increasing lysine levels in diets were used. The feed conversion data from selected works were converted to a common basis and submitted to regression analyzes considering the lysine level as independent variable by the exponential, quadratic model and Linear Response Plateau (LRP). Based on exponential equation was calculated the variation of feed cost per $\mathrm{kg}$ of weight gain. The cost of basal diet (without Llysine) was considered as $R \$ 0.70 / \mathrm{kg}$, and assigned the value of $2.80(\mathrm{~g} / \mathrm{g})$ for the FC desired. The optimal total lysine level appropriate to optimize performance, recommended by quadratic model was $1.036 \%$, while the optimum level recommen- 
PORTELA ETAL.

ded to reduce feed costs and increase the margin was $0.980 \%$, when the cost of L-lysine $\mathrm{HCl} \mathrm{kg}$ $(78.5 \%)$ was $\mathrm{R} \$ 4.71$. On the other hand, when the $\mathrm{kg}$ of L-lysine $(78.5 \%)$ increased to $R \$ 7.85$, the recommended level was reduced to $0.880 \%$. To estimate the feed cost per $\mathrm{kg}$ of weight gain for growing/finishing pigs, an equation was proposed.

\section{INTRODUÇÃO}

Dentre os aminoácidos essenciais, a lisina é o primeiro limitante para o desenvolvimento de suínos em terminação nas rações à base de milho e farelo de soja (NRC, 1998; Rostagno et al., 2011). Deste modo, para que o nutricionista formule rações capazes de suprir as necessidades de lisina, com níveis proteicos adequados, é imprescindível a utilização de uma fonte sintética deste aminoácido, como estratégia para reduzir os níveis de farelo de soja, principal componente do custo das rações.

A maioria dos estudos tradicionalmente conduzidos não considera análises de custos, baseando-se apenas na descrição quantitativa de variáveis produtivas em resposta ao aumento da concentração de lisina nas rações, sendo as recomendações correspondentes a concentrações capazes de maximizar ou otimizar o ganho de peso, a conversão alimentar, o rendimento de carcaça, entre outras variáveis (Owens e Pettigrew, 1989; Sakomura e Rostagno, 2007).

De acordo com Pack et al. (2003), os estudos para quantificar as exigências de nutrientes essenciais, incluindo aminoácidos, deveriam considerar variáveis de relevância econômica nas análises, pois as concentrações de aminoácidos que proporcionam máxima eficiência produtiva, na maioria das vezes, não coincidem com aquelas necessárias para alcançar a máxima lucratividade, principal objetivo dos produtores. Assim, o termo exigência, considerando como concentração fixa de aminoácidos é utilizado indevidamente, uma vez que as concentrações nas rações deverão variar de acordo com os preços dos insumos, que estão sujeitos a frequentes oscilações dependendo das condições do mercado.

Outro argumento baseia-se na variação individual existente dentro de uma população de animais, uma vez que ao definir uma concentração fixa visando atender as necessidades de um lote, existe a possibilidade de sobre-fornecimento para aqueles indivíduos com menor potencial e subfornecimento de nutrientes para aqueles indivíduos com maior potencial de resposta (Siqueira et al., 2009). Com base nisso, Sakomura e Rostagno (2007) relataram que o mais importante é entender como uma população ou lote de animais responde ao acréscimo na concentração de um aminoácido na ração, identificando-se a região crítica das curvas resposta, o que possibilita a associação de variáveis econômicas para a definição dos níveis ótimos de aminoácidos nas rações.

O procedimento estatístico utilizado para comparar informações provenientes de diferentes condições com resultados de estudos distintos, mas relacionados, definido por Glass (1976) como metanálise, tem sido adotado constantemente nas áreas de ciências. A metanálise consiste em um conjunto de métodos que busca sintetizar as informações quantitativas de estudos independentes, com o objetivo de explicar a variação dos efeitos obtidos nesses estudos. De acordo com Luiz (2002), a metanálise consiste em extrair informações de dados preexistentes e através da combinação dos resultados e aplicação de técnicas estatísticas, sintetiza-se suas conclusões ou até mesmo cria-se uma nova conclusão.

Diante do exposto, realizou-se este trabalho com o objetivo de estimar níveis de lisina em rações de suínos em terminação, para otimizar o desempenho e reduzir os custos com alimentação utilizando a técnica da metanálise, e propor um modelo para simular o custo com alimentação de acordo com o nível de lisina da ração. 


\section{LISINA PARA SUÍNOS: METANÁLISE PARA OTIMIZARO DESEMPENHO}

\section{MATERIALE MÉTODOS}

Foi realizada uma revisão na literatura brasileira e identificados trabalhos que avaliaram rações contendo níveis crescentes de lisina (Lys) para suínos na fase de terminação. Os trabalhos foram extraídos da Revista Brasileira de Zootecnia, Arquivo Brasileiro de Medicina Veterinária e Zootecnia, Revista Ciência e Agrotecnologia e Acta Scientiarum. Os artigos foram publicados entre os anos de 2000 e 2011 , e do total de 28 referências identificadas, 16 foram selecionadas para constituir o banco de dados.

Os trabalhos selecionados para compor o banco de dados (tabela I) atenderam os seguintes critérios de inclusão: 1-Estudos realizados no Brasil, publicados entre os anos de 2000 e 2011, que utilizaram suínos com peso entre 50 e $111 \mathrm{~kg}$; 2- Estudos de natureza quantitativa, instalados em delineamento inteiramente casualizado (DIC) ou blocos casualizados (DBC) com no mínimo três repetições; 3- Estudos que utilizaram rações com no mínimo três níveis de Lys, formuladas com base em níveis de aminoácidos totais ou digestíveis; 4Avaliaram variáveis de desempenho (consumo de ração, CR; ganho de peso, GP e conversão alimentar, CA) em resposta aos níveis de Lys nas rações.

Quando as rações foram apresentadas com base em Lys digestível, os níveis testados foram convertidos para Lys total considerando os coeficientes de digestibilidade das rações, calculados à partir da composição das rações experimentais apresentada em cada trabalho.

Os dados médios de CA, apresentados em cada um dos trabalhos selecionados, foram tabulados e transformados para uma base comum, possibilitando que a metanálise fosse realizada. Essa transformação consistiu em expressar os dados de cada trabalho como respostas relativas, atribuindo-se o valor de $100 \%$ à melhor resposta de $\mathrm{CA}$ em função do nível de Lys, expressando as demais respostas como porcentagens da melhor resposta, conforme proposto por Pack et al. (2003).

O conjunto de dados transformados de CA em resposta aos níveis crescentes de Lys, foi submetido a um teste de normalidade (Cramer Von-Mises), e atendida esta pressuposição foram realizadas análises de regressão considerando o nível de Lys como variável independente, por meio dos seguintes modelos:

$$
\begin{gathered}
\text { 1- Linear Response Plateau (LRP): } \\
\mathrm{Y}_{\mathrm{i}}=\mathrm{L}+\mathrm{U}\left(\mathrm{R}-\mathrm{Lys}_{\mathrm{i}}\right)+e_{\mathrm{i}}, \mathrm{i}=1,2 \ldots \mathrm{n}_{1}, \mathrm{n}_{1}+1, \ldots, \mathrm{n}
\end{gathered}
$$

em que:

$\left(\mathrm{R}-\mathrm{Lys}_{\mathrm{i}}\right)=0$ para $\mathrm{i}=\mathrm{n}_{1}+1$;

$\mathrm{n}_{1}=$ número de observações até o ponto de quebra;

$\mathrm{n}=$ número de pares de observações;

$Y_{i}=C$ A relativa estimada para o i-ésimo nível de Lys das rações;

Lys = nível de lisina das rações;

$\mathrm{L}=\mathrm{CA}$ relativa estimada no platô;

$\mathrm{U}=$ inclinação da reta ascendente;

$\mathrm{R}=$ nível de Lys estimado pelo ponto de quebra;

$e=$ erro ou desvio associado à distância entre o valores de CA relativa observados e os valores estimados pelas equações.

2-Polinomial quadrático: $Y_{i}=\beta_{0}+\beta_{1}$ Lys $_{i}+\beta_{2}$ Lys $_{i}^{2}+e_{i}$

em que:

$\beta_{0}=$ constante da regressão ou intercepto;

$\beta_{1}=$ parâmetro da regressão para o componente linear e;

$\beta_{2}=$ parâmetro da regressão para o componente quadrático.

3-Exponencial:

$\mathrm{Y}_{\mathrm{i}}=\mathrm{A}+\mathrm{B}\left(1-\mathrm{e}^{-\mathrm{C}(\text { Lysi }-\mathrm{D})}\right)+e_{\mathrm{i}}$

em que:

$A=$ resposta de $C A$ relativa estimada para a ração contendo o menor nível de Lys (Basal);

$\mathrm{B}=$ diferença estimada entre a mínima e a máxima resposta obtida com a suplementação de Lys;

$\mathrm{C}=$ coeficiente de inclinação da curva;

$\mathrm{D}=$ nível de Lys da ração basal, sendo $\mathrm{e}=$ base do logaritmo neperiano $(2,718282)$. 
PORTELA ET AL.

Tabela I. Trabalhos selecionados para compor o banco de dados utilizado na metanálise. (Papers selected to compose the database used in the meta-analysis).

\begin{tabular}{|c|c|c|c|c|c|c|c|c|}
\hline Referência/Linhagem & Sexo & $\mathrm{N}$ & $\begin{array}{l}\text { AT } \\
(\%)\end{array}$ & $\mathrm{PI}(\mathrm{kg})$ & IP & PB (\%) & Energia & $\begin{array}{l}\text { Rec. } \\
(\%)\end{array}$ \\
\hline \multicolumn{9}{|l|}{ Gomes et al. (2000) } \\
\hline ME (LW, LD,DR, HS, LB, PT) & $\mathrm{MC}$ & 3 & $0,90-1,15$ & 56,00 & $\mathrm{M}, \mathrm{FS}$ & 18,62 & ED 3353 & 0,90 \\
\hline ME (LW, PT, YS) & $\mathrm{MC}$ & 3 & $0,85-0,95$ & 86,00 & $\mathrm{M}, \mathrm{FS}$ & 17,68 & ED 3353 & 0,90 \\
\hline \multicolumn{9}{|l|}{ Fontes et al. (2000) } \\
\hline ME (HS, LB, PT) & $\mathrm{F}$ & 5 & $0,75-1,15$ & 61,20 & $\mathrm{M}, \mathrm{FS}$ & 15,80 & ED 3315 & 1,00 \\
\hline \multicolumn{9}{|l|}{ Moreira et al. (2002) } \\
\hline ME (Ag PIC C15; Ag 400, 405) & ) $\mathrm{MC}$ & 4 & $0,60-1,05$ & 53,40 & $\mathrm{M}, \mathrm{FS}$ & 11,60 & ED 3355 & $>1,05$ \\
\hline $\mathrm{ME}(\mathrm{LD}, \mathrm{LW}, \mathrm{DR})$ & MC & 4 & $0,60-1,05$ & 50,40 & $\mathrm{M}, \mathrm{FS}$ & 11,60 & ED 3355 & $<0,60$ \\
\hline \multicolumn{9}{|l|}{ Oliveira et al. (2003a) } \\
\hline Híbridos comerciais & MC & 5 & $0,50-0,90$ & 95,70 & $\mathrm{M}, \mathrm{FS}, \mathrm{GM}$ & 13,70 & ED 3367 & 0,76 \\
\hline \multicolumn{9}{|l|}{ Oliveira et al. (2003b) } \\
\hline Híbridos comerciais & MC & 4 & $0,50-0,80$ & 110,10 & $\mathrm{M}, \mathrm{FS}, \mathrm{GM}$ & 13,70 & ED 3367 & 0,80 \\
\hline \multicolumn{9}{|l|}{ Kill et al. (2003) } \\
\hline Híbridos comerciais & $\mathrm{F}$ & 4 & $0,80-1,10$ & 66,30 & $\mathrm{M}, \mathrm{FS}$ & 17,71 & EM 3300 & 1,00 \\
\hline \multicolumn{9}{|l|}{ Moreira et al. (2004) } \\
\hline Comum & $\mathrm{MC}$ & 4 & $0,60-1,05$ & 62,50 & $\mathrm{M}, \mathrm{FS}$ & 11,07 & ED 3347 & $<0,60$ \\
\hline Melhorado & $\mathrm{MC}$ & 4 & $0,60-1,05$ & 59,32 & $\mathrm{M}, \mathrm{FS}$ & 11,07 & ED 3347 & $<0,60$ \\
\hline \multicolumn{9}{|l|}{ Arouca et al. (2004) } \\
\hline Ag PIC 412xC-22 (HC) & $\mathrm{MC}$ & 5 & $0,50-0,90$ & 95,39 & $\mathrm{M}, \mathrm{FS}, \mathrm{GM}$ & 13,50 & EM 3204 & 0,76 \\
\hline \multicolumn{9}{|l|}{ Arouca et al. (2005) } \\
\hline LD, LW & MC & 5 & $0,50-0,90$ & 96,19 & $\mathrm{M}, \mathrm{FS}, \mathrm{GM}$ & 13,50 & EM 3204 & 0,61 \\
\hline \multicolumn{9}{|l|}{ Arouca et al. (2007) } \\
\hline Ag PIC 412xC-22 (HC) & $\mathrm{MC}$ & 5 & $0,50-0,90$ & 95,39 & $\mathrm{M}, \mathrm{FS}, \mathrm{GM}$ & 13,50 & EM 3204 & 0,72 \\
\hline \multicolumn{9}{|l|}{ Rossoni (2007) } \\
\hline Híbridos comerciais & $\mathrm{F}$ & 5 & $0,73-1,13^{*}$ & 61,45 & $\mathrm{M}, \mathrm{FS}$ & 17,47 & EM 3273 & $0,99^{*}$ \\
\hline \multicolumn{9}{|l|}{ Abreu et al. (2007) } \\
\hline Híbridos comerciais & $\mathrm{MC}$ & 4 & $0,70-1,00^{*}$ & 60,43 & $\mathrm{M}, \mathrm{FS}$ & 16,73 & EM 3250 & $0,94^{*}$ \\
\hline \multicolumn{9}{|l|}{ Silva et al. (2009) } \\
\hline Híbridos comerciais & $\mathrm{MC}$ & 4 & $0,75-1,05^{\star}$ & 59,84 & M, FS & 17,14 & EM 3322 & $0,96^{*}$ \\
\hline \multicolumn{9}{|l|}{ Almeida et al. (2010) } \\
\hline TOPPxC-40(HC) & $\mathrm{MC}, \mathrm{F}$ & 5 & $0,68-1,08^{*}$ & 90,20 & $\mathrm{M}, \mathrm{FS}$ & 11,07 & EM 3212 & $0,68^{*}$ \\
\hline \multicolumn{9}{|l|}{ Souza et al. (2011) } \\
\hline Híbridos comerciais & MC & 4 & $0,80-1,10^{*}$ & 90,19 & $\mathrm{M}, \mathrm{FS}$ & 17,42 & EM 3230 & $0,80^{*}$ \\
\hline \multicolumn{9}{|l|}{ Santos et al. (2011) } \\
\hline Híbridos comerciais & $\mathrm{MC}$ & 5 & $0,54-0,948^{*}$ & 95,55 & M,FS,GM & 15,90 & ED 3400 & $0,817^{*}$ \\
\hline $\begin{array}{l}\mathrm{N}=\mathrm{N} \text { o de níveis; } \mathrm{AT}=\text { amplitude te } \\
\text { proteína bruta da ração (\%); } \mathrm{ED}= \\
\mathrm{ME}=\text { mestiços; } \mathrm{HC}=\text { híbridos com } \\
\mathrm{LB}=\text { Landrace Belga; } \mathrm{PT}=\text { Pietrain } \\
\mathrm{FS}=\text { farelo de soja; } \mathrm{GM}=\text { glúten d } \\
\text { *Lisina digestível }(\%) .\end{array}$ & $\begin{array}{l}\text { estada; } \\
=\text { enerc } \\
\text { nerciais } \\
\mathrm{n} ; \mathrm{YS}= \\
\text { de milho }\end{array}$ & & $\begin{array}{l}\text { peso inicial } \\
\text { igestível; Re } \\
=\text { Large Whit } \\
\text { shire. } \mathrm{MC}=\mathrm{m} \\
\mathrm{l}=\text { energia } \mathrm{m}\end{array}$ & $\begin{array}{l}\text { ite; } L D=L \\
\text { machos } \\
\text { netaboliz }\end{array}$ & $\begin{array}{l}=\text { ingrediente } \\
\text { el recomend } \\
\text { Landrace; DF } \\
\text { castrados; F } \\
\text { zável. }\end{array}$ & $\begin{array}{l}\mathrm{R}=\text { Duroc } \\
=\text { fêmeas }\end{array}$ & $\begin{array}{l}\text { bais da raçã } \\
\text { ina total; \% } \\
\text { c; HS= Ham } \\
\text { s. } M=\text { milho }\end{array}$ & $\begin{array}{l}\text { o; } P B= \\
\text { oshire; } \\
\text { noído; }\end{array}$ \\
\hline
\end{tabular}

Archivos de zootecnia vol. 63, núm. 243, p. 422. 


\section{LISINA PARA SUÍNOS: METANÁLISE PARA OTIMIZARO DESEMPENHO}

Para verificar a qualidade do ajuste dos diferentes modelos, foram considerados os valores dos quadrados médios dos desvios (QM desvios), as significâncias dos testes $\mathrm{F}$ e os coeficientes de determinação $\left(\mathrm{R}^{2}=\mathrm{SQ}\right.$ modelo/SQ total).

As recomendações dos níveis de Lys para otimizar o desempenho de suínos em terminação foram obtidas por diferentes métodos, considerando cada um dos modelos de regressão ajustados, conforme apresentado por Sakomura e Rostagno (2007). Pelo LRP estas estimativas foram obtidas pelo encontro da reta ascendente com o platô. Para o modelo polinomial quadrático, as estimativas foram obtidas igualando-se primeira derivada da equação a zero $\left(2 \beta_{2} / \beta_{1}\right)$. Os níveis ótimos estimados com o uso do modelo exponencial foram obtidos considerando $95 \%$ da resposta assintótica (melhores respostas) por meio da expressão: $(\ln 0,05) / \mathrm{C}+\mathrm{D}$.

As recomendações dos níveis de Lys adequadas para reduzir os custos com alimentação foram obtidas com base na metodologia proposta por Pack et al. (2003) simulando-se diferentes preços da L-lisina $\mathrm{HCl}$. A variação no custo com alimentação $(\Delta \mathrm{R} \$)$, proporcionada pela adição de níveis crescentes de Lys foi calculada pela expressão:

$$
\Delta R \$=C R B+\left((\text { CULys }- \text { CURB })^{*} N S L y s\right)
$$

em que:

$\mathrm{CRB}=$ custo do $\mathrm{kg}$ da ração basal sem adição de L-lisina $\mathrm{HCl}(\mathrm{R} \$)$;

CULys = custo por unidade de Lys suplementar $(\mathrm{R} \$$ ) (custo do $\mathrm{kg}$ de L-lisina $\mathrm{HCl} / 100$ );

CURB= custo unitário da ração basal $(\mathrm{R} \$)$ (custo do kg da ração basal/100);

NSLys= acréscimo na concentração de Lys (\%) proporcionado pela suplementação de L-lisina $\mathrm{HCl}$ na ração basal $\left(\right.$ Lys $\left._{i}-\mathrm{D}\right)$.

Para estimar os valores absolutos de CA à partir da equação ajustada para os valores relativos $(\mathrm{CA} \%)$, atribuiu-se um máximo valor de CA de acordo com o potencial do rebanho $(2,80 \mathrm{~g} / \mathrm{g})$.

Multiplicando-se os valores de CA (g/g) estimados pela equação exponencial em cada nível de Lys avaliado, pelo custo do alimento calculado para cada nível de suplementação $(\Delta \mathrm{R} \$)$, foi obtida a variação do custo com alimentação/kg de GP $(\Delta \mathrm{R} \$ / \mathrm{kgGP})$, por unidade de Lys suplementar.

A margem por $\mathrm{kg}$ de suíno produzido (M) em cada nível de suplementação de Lys nas rações foi calculada pela expressão:

$$
M=P V S-(R \$ / k g G P)
$$

em que:

$\mathrm{PVS}=$ preço de venda do $\mathrm{kg}$ de suíno vivo $(\mathrm{R} \$)$; $(\mathrm{R} \$ / \mathrm{kgGP})=$ custo com alimentação por $\mathrm{kg}$ de suíno produzido $(\mathrm{R} \$)$ para cada nível de Lys na ração.

Os níveis de Lys estimados para reduzir os custos com alimentação foram definidos como sendo aqueles que proporcionaram os menores custos com alimentação por $\mathrm{kg}$ de GP e maior margem.

Os ajustes dos diferentes modelos de regressão foram realizados com o auxílio do software SAS 9.0 (Statistical Analysis System).

\section{RESULTADOSEDISCUSSÃO}

A maioria dos estudos desenvolvidos para determinar exigências de aminoácidos para suínos tem sido do tipo dose-resposta e a principal técnica de análise estatística utilizada para estimar os níveis ótimos de um nutriente é a análise de regressão, destacando-se o modelo polinomial quadrático, por ser de fácil manipulação e determinar um único valor de exigência de maneira simples e rápida. Entretanto este modelo tem sido criticado por vários autores (Euclydes e Rostagno, 2001; Lamberson e Firman, 2002; Sakomura e Rostagno, 2007; Siqueira et al., 2009) principalmente pela possibilidade de superestimar os valores das exigências.

Outro modelo que tem sido utilizado é o 
PORTELA ET AL.

LRP, no entanto é criticado por proporcionar uma interpretação simplificada da curva-resposta, assumindo que a utilização do nutriente limitante é constante até que sua exigência seja suprida e que não há respostas adicionais no desempenho acima deste ponto, havendo a possibilidade de subestimativas (Robbins et al., 1979; Pack et al., 2003).

Diante disso, alguns autores (Sarmento et al., 2003; Pack et al., 2003; Sakomura e Rostagno, 2007; Siqueira et al., 2009; Siqueira et al., 2011), têm proposto a utilização de modelos de natureza não linear, como o exponencial, que descreve de maneira detalhada a resposta animal, permitindo a associação de variáveis econômicas na determinação do níveis ótimos.

Os modelos LRP, polinomial quadrático e exponencial se ajustaram aos dados de conversão alimentar relativa (CA, \%), proporcionando coeficientes de determinação $\left(\mathrm{R}^{2}\right)$ de 0,$55 ; 0,58$ e 0,55 , respectivamente (tabela II). Estes valores podem ser explicados com base no grande volume de dados $(n=70)$, obtidos de estudos com suínos de diferentes linhagens, mantidos sob diferentes condições experimentais com pesos entre 50,4 e 110,1 kg.

Do ponto de vista estatístico, o modelo polinomial quadrático foi o que melhor se ajustou aos dados apresentando o menor quadrado médio do resíduo (QMRes=
9,9968) e o maior coeficiente de determinação $\left(\mathrm{R}^{2}=0,58\right)$, seguido pelo LRP (QMRes= 10,$\left.5819 ; R^{2}=0,55\right)$ e pelo exponencial $\left(\mathrm{QMRes}=10,7442 ; \mathrm{R}^{2}=0,55\right)$.

As estimativas dos níveis ótimos de lisina apresentadas na tabela II variaram de acordo com o modelo utilizado, o que é perfeitamente explicável, pois cada modelo estima o nível ótimo de acordo com diferentes critérios. Pelo modelo LRP obteve-se a menor estimativa $(0,931 \%)$, enquanto que pelo exponencial, considerando $95 \%$ da resposta assintótica, obteve-se a maior $(1,122 \%)$, sendo uma estimativa intermediária obtida pelo modelo quadrático $(1,036$

$\%$ ) (figura 1). Estes resultados ilustram a dificuldade em se eleger uma dose ótima em ensaios dose-resposta, pois utilizando o mesmo conjunto de dados é possível obter diversas recomendações para uma mesma variável.

O nível ótimo de lisina total de 1,036\%, equivalente a $0,901 \%$ de lisina digestível, estimado pelo modelo quadrático para otimizar a CA de suínos em terminação foi próximo às recomendações de Fontes et al. (2000) de 1,00\% (0,90\% de lisina digestível) e Abreu et al. (2007) de 1,075\% de lisina total $(0,94 \%$ de lisina digestível) em rações para suínos machos castrados de 60 a $95 \mathrm{~kg}$, ambas obtidas pelo modelo quadrático. Entretanto foi superior à recomendação de Santos et al. (2011) de 0,89\% de lisina total $(0,82 \%$ de lisina digestível) para suínos

Tabela II. Equações ajustadas para a conversão alimentar relativa (CA;\%) em função dos níveis de lisina total das rações (Lys;\%). (Equations adjusted for relative feed conversion (CA;\%) as function of total lysine levels (Lys;\%) in diets).

\begin{tabular}{|c|c|c|c|c|c|}
\hline Modelos & Equações & $\mathrm{p}>\mathrm{F}^{1}$ & QMRes $^{2}$ & $\mathrm{R}^{2(3)}$ & $\mathrm{NLE}^{4}(\%)$ \\
\hline LRP & $C A=97,378+28,723$ (0,931-Lys) & $<0,001$ & 10,5819 & 0,55 & 0,931 \\
\hline Quadrático & $\mathrm{CA}=44,751+101,532$ Lys-49,006 Lys $^{2}$ & $<0,001$ & 9,9968 & 0,58 & 1,036 \\
\hline Exponencial & $C A=83,079+15,096\left(1-e^{-4,813(L y s-0,500)}\right)$ & $<0,001$ & 10,7442 & 0,55 & 1,122 \\
\hline
\end{tabular}

${ }^{1}$ Significância do teste "F"para o modelo; ${ }^{2}$ Quadrado médio do resíduo; ${ }^{3}$ Coeficiente de determinação $\left(\mathrm{R}^{2}=\right.$ SQModelo/SQTotal); " ${ }^{4} \mathrm{Nível}$ ótimo de lisina total estimado.

Archivos de zootecnia vol. 63, núm. 243, p. 424. 


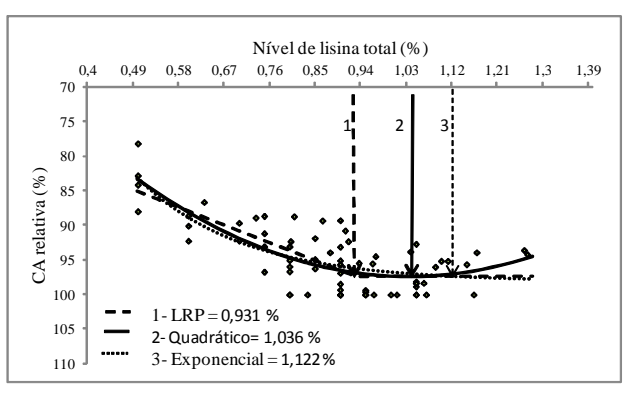

Figura 1. Representação gráfica das equações ajustadas e dos níveis recomendados por diferentes modelos para a conversão alimentar relativa $(C A ; \%)(n=70)$. (Graphical representation of equations adjusted accord to different models $(\mathrm{CA} ; \%)$ as function of total lysine levels (Lys; \%) in diets $(n=70)$ ).

machos castrados de 95 a $125 \mathrm{~kg}$, obtida pelo mesmo modelo.

Pelo modelo LRP, o nível ótimo de lisina total foi de $0,931 \%(0,810 \%$ de lisina digestível), sendo superior a recomendação de Oliveira et al. (2003a) de 0,79\% (0,716\% de lisina digestível) para suínos de 95 a 110 $\mathrm{kg}$, e inferior a recomendação de Silva et al. (2009) de 1,083\% (0,96\% de lisina digestível) para suínos de 60 aos $95 \mathrm{~kg}$, ambas obtidas com o uso de modelo LRP.

No presente estudo, a maior estimativa do nível de lisina adequado para otimizar a CA foi de $1,122 \%(0,976 \%$ de lisina digestível), obtida pelo modelo exponencial, sendo possivelmente uma superestimativa da exigência de suínos em crescimento/ terminação, por ser marginal ao maior nível testado $(1,270 \%)$. Entretanto, este modelo é recomendado por descrever de forma detalhada os pequenos acréscimos no desempenho animal decorrentes dos níveis nutricionais da dieta, o que o torna de grande utilidade quando o interesse é determinar o nível ótimo de nutrientes do ponto de vista econômico (Pack et al., 2003; Sakomura e Rostagno, 2007; Siqueira et al., 2009; Siqueira et al., 2011).

Para a realização dos cálculos dos níveis ótimos de lisina total do ponto de vista econômico, o custo da ração basal (isenta de L-lisina $\mathrm{HCl}$ ) foi considerado como sendo $\mathrm{R} \$ 0,70 / \mathrm{kg}$, e a resposta de CA desejada, de acordo com o potencial genético do rebanho, de $2,80(\mathrm{~g} / \mathrm{g})$.

Considerando que a L-lisina $\mathrm{HCl}$ utilizada nos estudos continha 78,5\% de lisina, atribuindo-se o preço de $\mathrm{R} \$ * 4,71 / \mathrm{kg}$ para este insumo, o kg de lisina custará $\mathrm{R} \$ 6,00$ $(4,71 / 0,785=6,00)$. Assim, o menor custo com alimentação por kg de GP foi estimado em R \$ $2,101(\mathrm{R} \$ / \mathrm{kgGP}=(0,70+(((6 / 100)-(0,70 /$ $\left.\left.\left.100))^{*} 0,48\right)\right)^{*} 2,896=2,101\right)$. Assumindo um preço de venda do suíno vivo de R \$ 2,50 (Suinocultura industrial, 2012) a margem por $\mathrm{kg}$ de suíno produzido foi de $\mathrm{R} \$ 0,399$ $(\mathrm{M}=2,50-2,101=0,399)$, correspondendo ao nível de lisina total estimado em $0,980 \%$ $(0,852 \%$ de lisina digestível), capaz de proporcionar menor custo com alimentação e maior margem.

Entretanto, tendo em vista as oscilações no preço de mercado dos insumos utilizados na nutrição de suínos, se o custo da Llisina $\mathrm{HCl}$ aumentar para $\mathrm{R} \$ 7,85 / \mathrm{kg}$ (custo da lisina $=\mathrm{R} \$ 10,00 / \mathrm{kg}$ ), o menor custo com alimentação por $\mathrm{kg}$ de GP será de R \$ 2,150 $(\mathrm{R} \$ / \mathrm{kgGP}=(0,70+(((10 / 100)-(0,70 / 100) *$ $\left.0,38))^{*} 2,924=2,150\right)$, e a margem reduzirá para $\mathrm{R} \$ 0,35(\mathrm{M}=2,50-2,150=0,35)$, correspondendo ao nível de lisina estimado em $0,880 \%(0,765 \%$ de lisina digestível $)$ (figura 2).

Para as condições simuladas no presente estudo, em que considerou-se apenas variações no preço da L-lisina $\mathrm{HCl}$, mantendo-se o preço dos demais ingredientes constante (ração basal $=\mathrm{R} \$ 0,70 / \mathrm{kg}$ ), um aumento de $66,6 \%$ no custo do aminoácido (de 4,71 para $\mathrm{R} \$ 7,85$ ), gerou a necessidade de redução no nível de lisina da ração em $10,2 \%$ (de 0,980 para $0,880 \%$ ) para que seja possível produzir com mínimo custo, que mesmo assim aumentou em 2,33\% (de 2,101 para $\mathrm{R} \$ 2,15$ ) ocasionando uma redução de

$* 1 \mathrm{R} \$=0,485$ US $\$$ 


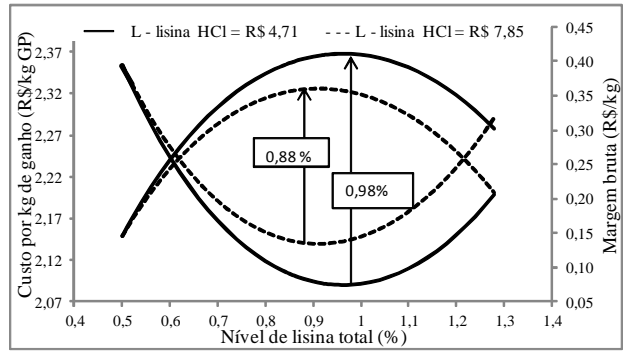

Figura 2. Representação gráfica da variação do custo com alimentação e da margem bruta por $\mathrm{kg}$ de suíno produzido em função dos níveis de lisina total (\%) da ração e do preço da L-lisina $\mathrm{HCl}(78,5 \%)$. (Graphical representation of variation in feed cost and gross margin per kg of swine produced accord to the total lysine levels in diets (\%), depending of price of L-lysine $\mathrm{HCl}(78.5 \%))$.

$12,3 \%$ na margem (de 0,399 para $\mathrm{R} \$ 0,35$ ).

Estes resultados ilustram o impacto da variação no custo da L-lisina $\mathrm{HCl}$, normalmente incluída em pequenas concentrações nas rações $(0,1$ a $0,3 \%)$, sobre o custo com alimentação por $\mathrm{kg}$ de suíno produzido, revelando que quando o preço da L-lisina $\mathrm{HCl}$ aumenta, para que o produtor consiga produzir com mínimo custo é necessário que o nível de lisina da ração seja reduzido (figura 2).

Considerando que, apesar das críticas, o modelo polinomial quadrático apresentou melhor ajuste do ponto de vista estatístico e gerou uma estimativa intermediária àquelas obtidas pelos modelos LRP e exponencial, recomenda-se o nível de lisina total de $1,036 \%(0,901 \%$ de lisina digestível) para otimizar o desempenho de suínos na fase de terminação.

Contudo, este estudo demonstrou que o conceito de exigência tratado como níveis fixos de nutrientes capazes de proporcionar o máximo desempenho pode ser questionável, corroborando os relatos de Pack et al. (2003), pois diante de oscilações nos preços dos insumos é inevitável que haja flexibilidade nos níveis nutricionais das rações para que se atinja o mínimo custo com alimentação e conseqüentemente máxima lucratividade.

Diante disso, para simular o custo com alimentação por $\mathrm{kg}$ de GP de suínos em terminação, de acordo com o nível de lisina, propõe-se a equação:

$\mathrm{R} \$ / \mathrm{kgGP}=\mathrm{CRB}+\left((\mathrm{CLys} / 100)-(\mathrm{CRB} / 100)^{*}\right.$ NSLys $)^{*}(C A D * 100 /(83,079+15,096 *(1-$ $\left.\left.2,71828^{-4,813^{*}(\text { Lys }-0,50)}\right)\right)$

em que:

$\mathrm{R} \$ / \mathrm{kg} \mathrm{GP}=$ custo $(\mathrm{R} \$)$ por $\mathrm{kg}$ de suíno produzido; $C R B=$ custo $(R \$)$ do $\mathrm{kg}$ da ração isenta de L-lisina $\mathrm{HCl}$ (basal);

CLys $=$ custo $(\mathrm{R} \$)$ por $\mathrm{kg}$ de lisina suplementar; NSLys= acréscimo na concentração de lisina (\%) proporcionado pela suplementação de L-lisina $\mathrm{HCl}$ na ração basal;

$\mathrm{CAD}=$ conversão alimentar desejada de acordo com o potencial genético do rebanho $(\mathrm{g} / \mathrm{g})$;

Lys= nível de lisina total a ser utilizado na ração (\%).

\section{CONCLUSÕES}

O nível de lisina total em rações de suínos em terminação recomendado para otimizar o desempenho, estimado pelo modelo quadrático, é $1,036 \%$, correspondendo a 0,901 $\%$ de lisina digestível.

O nível ótimo de lisina total estimado para reduzir os custos com alimentação e aumentar a margem foi de $0,980 \%(0,852 \%$ de lisina digestível) quando a L-lisina $\mathrm{HCl}$ $(78,5 \%)$ custou $\mathrm{R} \$ 4,71 / \mathrm{kg}$. Por outro lado quando a L- lisina $\mathrm{HCl}(78,5 \%)$ aumentou para $\mathrm{R} \$ 7,85 / \mathrm{kg}$, o nível ótimo estimado reduziu para $0,880 \%(0,765 \%$ de lisina digestível).

Para simular o custo com alimentação por kg de GP de suínos em terminação, de acordo com os níveis e preço da Lisina, propõe-se a equação:

$\mathrm{R} \$ / \mathrm{kgGP}=\mathrm{CRB}+\left((\mathrm{CLys} / 100)-(\mathrm{CRB} / 100)^{*}\right.$ NSLys $) *(C A D * 100 /(83,079+15,096 *(1-$ $2,71828^{\left.-4,813^{*}(\text { Lys-0,50) })\right)}$ 


\section{LISINA PARA SUÍNOS: METANÁLISE PARA OTIMIZARO DESEMPENHO}

em que:

$\mathrm{R} \$ / \mathrm{kg} \mathrm{GP}=$ custo $(\mathrm{R} \$)$ por kg de suíno produzido;

$\mathrm{CRB}=$ custo $(\mathrm{R} \$)$ do $\mathrm{kg}$ da ração isenta de L-lisina $\mathrm{HCl}$ (basal);

CLys= custo $(R \$)$ por $\mathrm{kg}$ de lisina suplementar;

NSLys= acréscimo na concentração de lisina (\%) proporcionado pela suplementação de L-lisina $\mathrm{HCl}$ na ração basal;

$\mathrm{CAD}=$ conversão alimentar desejada de acordo com o potencial genético do rebanho $(\mathrm{g} / \mathrm{g})$;

Lys $=$ nível de lisina total a ser utilizado na ração (\%).

\section{BIBLIOGRAFIA}

Abreu, M.L.T.; Donzele, J.L.; Oliveira, R.F.M.; Oliveira, A.L.S.; Santos, F.A. e Pereira, A.A. 2007. Níveis de lisina digestível em rações, utilizando-se o conceito de proteína ideal, para suínos machos castrados de alto potencial genético para deposição de carne magra na carcaça dos 60 aos $95 \mathrm{~kg}$. Rev Bras Zootecn, 36: 54-61.

Almeida, E.C.; Fialho, E.T.; Rodrigues, P.B.; Zangeronimo, M.G.; Lima, J.A.F. and Fontes, D.O. 2010. Ractopamine and lysine levels on performance and carcass characteristics of finishing pigs. Rev Bras Zootecn, 39: 19611968.

Arouca, C.L.C.; Fontes, D.O.; Ferreira, W.M.; Silva M.A. e Pereira, F.A. 2004. Exigências de lisina, com base no conceito de proteína ideal, para suínos machos castrados, de 95 a $122 \mathrm{~kg}$, selecionados para deposição de carne magra. Arq Bras Med Vet Zoo, 56: 773-781.

Arouca, C.L.C.; Fontes, D.O.; Veloso, J.A.F.; Moreira, H.F.V. e Marinho, P.C. 2005. Exigências de lisina, com base no conceito de proteína ideal, para suínos machos castrados dos 96 aos $120 \mathrm{~kg}$, selecionados para eficiência de crescimento. Arq Bras Med Vet Zoo, 57: 104111.

Arouca, C.L.C.; Fontes, D.O.; Baião, N.C., Silva, M.A. e Silva, F.C.O. 2007. Exigências de lisina para suínos machos castrados selecionados geneticamente para deposição de carne magra na carcaça, dos 95 aos 122 kg. Ciênc Agrotec, 31: 531-5939.

Euclydes, R.F. e Rostagno, H.S. 2001. Estimativa dos níveis nutricionais via experimentos de
Por se tratar de uma equação de fácil utilização, que considera o custo da ração (CRB) e da fonte de lisina sintética (CLys), e considera ainda o nível de lisina a ser utilizado na ração (Lys) de acordo com o potencial genético do rebanho (CAD), pode ser considerada uma ferramenta de utilidade prática para que o produtor simule seus custos com alimentação auxiliando tomadas de decisão no cotidiano das granjas Brasileiras.

desempenho. Em: I Workshop Latino-Americano Ajinomoto Biolatina. Anais... Foz do Iguaçu. Brasil. pp. 77-88.

Fontes, D.O.; Donzele, J.L.; Ferreira, A.S.; Oliveira, R.F.M. e Júnior, C.A.G.G. 2000. Níveis de lisina para leitoas selecionadas geneticamente para deposição de carne magra, dos 60 aos $95 \mathrm{~kg}$. Rev Bras Zootecn, 29: 784-793.

Glass, G.V. 1976. Primary, secundary, and metaanalysis of research. Educ Res, 6: 3-8.

Gomes, F.E.; Fialho, E.T.; Lima, J.A.F.; Oliveira, A.I.G.; Bertechini, A.G. e Gonçalves, T.M. 2000. Planos de nutrição baseados em níveis de lisina para suínos de diferentes genótipos abatidos aos 80 e $100 \mathrm{~kg}$ de peso vivo. Ciênc Agrotec, 24: 479-489.

Kill, J.L.; Donzele, J.L.; Oliveira, R.F.M.; Ferreira, A.S.; Lopes, D.C.; Silva, F.C.O.S. e Silva, M.V.G.B. 2003. Planos de nutrição para leitoas com alto potencial genético para deposição de carne magra dos 65 aos $105 \mathrm{~kg}$. Rev Bras Zootecn, 32: 1330-1338.

Lamberson, W.R. and Firman, J.D. 2002. A comparison of quadratic versus segmented regression procedures for estimating nutrient requirements. Poultry Sci, 81: 481-484.

Luiz, A.J.B. 2002. Metanalise; Definição, aplicações e sinergia com dados espaciais. Cad Ciênc Tecnol, 19: 407-428.

Moreira, I.; Gasparotto, L.F.; Furlan, A.C.; Patrício, V.M.I. e Oliveira, G.C. 2002. Exigência de lisina para machos castrados de dois grupos genéticos de suínos na fase de terminação, com base no conceito de proteína ideal. Rev Bras Zootecn, 31: 96-103. 


\section{PORTELA ET AL.}

Moreira, I.; Kutschenko, M.; Furlan, A.C.; Murakami, A.E.; Martins, E.N. e Scapinello, C. 2004. Exigência de lisina para suínos em crescimento e terminação, alimentados com rações de baixo teor de proteína, formuladas de acordo com o conceito de proteína ideal. Acta Sci Anim Sci, 26: $537-542$.

NRC. 1998. National Research Council. Nutrient requirements of Swine. $10^{\text {th }}$ ed. National. Washington, DC. $189 \mathrm{pp}$.

Oliveira, A.L.S.; Donzele, J.L.; Oliveira, R.F.M.; Lopes, D.C.; Moita, A.M.S.; Silva, F.C.O. e Freitas, L.S. 2003a. Lisina em rações para suínos machos castrados selecionados para deposição de carne magra na carcaça dos 95 aos $110 \mathrm{~kg}$. Rev Bras Zootecn, 32: 337-343.

Oliveira, A.L.S.; Donzele, J.L.; Oliveira, R.F.M.; Ferreira, A.S.F.; Moita, A.M.S. e Generoso, R.A.R. 2003b. Lisina em rações para suínos machos castrados selecionados para deposição de carne magra na carcaça dos 110 aos 125 kg. Rev Bras Zootecn, 32: 150-155.

Owens, F.N. and Pettigrew, J.E. 1989. Subdividing amino acid requirements into portions for maintenance and growth. In: Friedman, M. (Ed.). Absorption and utilization of amino acids. CRC Press. Boca Raton. pp. 15-30.

Pack, M.; Hoehler, D. and Lemme, A. 2003. Economic assessment of amino acid responses in growing poultry. In: D'Mello, J.P.F. (Ed.). Amino acids in animal nutrition. CABI Publishing. Cambridge. pp. 459-483.

Rossoni, M.C. 2007. Níveis de lisina em rações para fêmeas suínas dos 15 aos $95 \mathrm{~kg}$. Tese (Doutorado). Universidade Federal de Viçosa. Viçosa-MG. 25 pp.

Rostagno, H.S.; Albino, L.F.T.; Donzele, J.L.; Gomes, P.C.; Oliveira, R.F.M.; Lopes, D.C.; Ferreira, A.S.; Barreto, S.L.T. e Euclides, R.F. 2011. Tabelas brasileiras para aves e suínos: composição de alimentos e exigências nutricionais. $3^{\underline{a}}$ ed. Universidade Federal de Viçosa. Viçosa, MG. Brasil. 252 pp.

Robbins, K.L.; Norton, H.W. and Baker, D.H. 1979.
Estimation of nutrient requirements from growth data. J Nutr, 109: 1710-1714.

Sakomura, N.K. e Rostagno, H.S. 2007. Métodos de pesquisa em nutrição de monogástricos. Funep. Jaboticabal. 283 pp.

Santos, F.A.; Donzele, J.L.; Silva, F.C.O.; Oliveira R.F.M.; Abreu, M.L.T.; Haese, D. e Lima, A.L. 2011. Níveis de treonina digestível em rações para suínos machos castrados de alto potencial genético na fase dos 95 aos $125 \mathrm{~kg}$. Rev Bras Zootecn, 40: 1038-1044.

Sarmento, J.L.R.; Torres, R.A. e Sousa, W.H. 2003. Comparação de modelos de regressão não-linear no ajuste da curva de crescimento de ovinos Santa Inês. 40 ${ }^{a}$ Reunião Anual da Sociedade Brasileira de Zootecnia. Anais... Santa Maria. 5 pp.

Silva, J.C.; Donzele, J.L.; Oliveira, R.F.M.; Ferreira, A.S.; Neto, A.M. e Paula, E. 2009. Influência do número de animais e tipo de comedouro na unidade experimental sobre as exigências nutricionais e composição de carcaça de suínos em terminação. Rev Bras Zootecn, 38: 10591067.

Siqueira, J.C.; Sakomura, N.K.; Nascimento, D.C.N. e Fernandes, J.B.K. 2009. Modelos matemáticos para estimar as exigências de lisina digestível para aves de corte ISA Label. Rev Bras Zootecn, 38: 1732-1737.

Siqueira, J.C.; Sakomura, N.K.; Dorigam, J.C.P.; Mendonça, G.G.; Costa, F.G.P.; Fernandes, J.B.K.; Dourado, L.R.B. e Nascimento, D.C.N. 2011. Níveis de lisina em rações de frangos de corte determinados com base em uma abordagem econômica. Rev Bras Zootecn, 40: 2178-2185.

Souza, E.O.; Haese, D.; Kill, J.L.; Haddade, I.R.; Lacerda, E.G.; Saraiva, A.; Silva, F.C.O. and Sobreiro, R.P. 2011. Digestible lysine levels in diets supplemented with ractopamine. Rev Bras Zootecn, 40: 2186-2191.

Suinocultura Industrial. 2012. Análise de mercado. <http://www.suinoculturaindustrial.com.br/ analise $>$ (10/05/2012). 